\title{
CONGRUENCE OF QUATERNIONIC SKEW-HERMITIAN MATRICES
}

\author{
David W. Lewis
}

It is well-known from the theory of hermitian forms, [S, ch.10, $\S 3]$ that two non-singular skew-hermitian forms over the real quaternions are isometric if and only if they have the same dimension. In matrix terms this means that any two invertible $n \times n$ skew-hermitian matrices, with entries from the real quaternions, are necessarily congruent. This result for quaternionic matrices must have been known for a very long time but it does not seem to appear explicitly in the literature. (It can, of course, be deduced from the unitary triangularization theorem for quaternionic matrices which was proved in [L] and [B]!) Hence we hope it is worthwhile to present the following elementary proof.

Let $\mathbf{H}$ denote the real quaternions, generated in the usual way by elements $i, j$ with $i^{2}=j^{2}=-1, i j=k=-j i$, etc. Let - be the standard involution on $\mathbf{H}$, so that if

$$
q=\alpha+\beta i+\gamma j+\delta k
$$

then

$$
\bar{q}=\alpha-\beta i-\gamma j-\delta k
$$

where $\alpha, \beta, \gamma$ and $\delta$ are real numbers.

An $n \times n$ matrix $A=\left(a_{l m}\right)$ with entries from $\mathbf{H}$ is said to be invertible if there exists an $n \times n$ matrix $\mathrm{C}$ with entries from $\mathbf{H}$ such that $A C=C A=I_{n}$, the $n \times n$ identity matrix.

An $n \times n$ matrix $A=\left(a_{l m}\right)$ with entries from $\mathbf{H}$ is said to be skew-hermitian if $\bar{A}^{t}=-A$, i.e. if $\bar{a}_{l m}=-a_{m l}$ for all $l$ and $m$.

Two $n \times n$ matrices $A$ and $B$ with entries from $\mathbf{H}$ are said to be congruent if there exists an invertible $n \times n$ matrix $P$ with entries 
from $\mathbf{H}$ such that $\bar{P}^{t} A P=B$. (Congruence is an equivalence relation on the set of all $n \times n$ skew-hermitian matrices. Note that $(\overline{P Q})^{t}=\bar{Q}^{t} \bar{P}^{t}$ for any $n \times n$ matrices $P$ and $Q$.)

Theorem Any two invertible $n \times n$ skew-hermitian matrices with entries from $\mathbf{H}$ are congruent.

Proof: It suffices to show that any invertible skew-hermitian $n \times n$ matrix $A$ is congruent to $i I_{n}$, the diagonal matrix with $i$ in each diagonal place. We will prove this statement by induction on $n$.

Let $n=1$ and $A=(a)$ be skew-hermitian, so that

$$
a=\beta i+\gamma j+\delta k
$$

for some $\beta, \gamma$ and $\delta$ in $\mathbf{R}$, not all zero. We seek $z \in \mathbf{H}$ such that $a=\bar{z} i z$. We will show that it is always possible to find $z \in \mathbf{H}$ of the form $z=r i+s j+t k$, where $r, s, t$ are real numbers, for which $a=\bar{z} i z$. Since

$$
\bar{z} i z=\left(r^{2}-s^{2}-t^{2}\right) i+2 r s j+2 r t k,
$$

we have equations

$$
\begin{aligned}
r^{2}-s^{2}-t^{2} & =\beta \\
2 r s & =\gamma \\
2 r t & =\delta .
\end{aligned}
$$

Equating norms on each side of the equality $a=\bar{z} i z$ yields another equation:

$$
\left(r^{2}+s^{2}+t^{2}\right)^{2}=\beta^{2}+\gamma^{2}+\delta^{2} .
$$

Taking the positive square root of this equation and adding the first equation above, we see that

$$
2 r^{2}=\beta+\sqrt{\left(\beta^{2}+\gamma^{2}+\delta^{2}\right)} .
$$

Now $\beta+\sqrt{\left(\beta^{2}+\gamma^{2}+\delta^{2}\right)} \geq 0$ and equals zero only if $\gamma=\delta=0$, and $\beta<0$. If $\gamma=\delta=0$ and $\beta<0$, we choose $r=t=0$, $s=\sqrt{(-\beta)}$. Otherwise, we can choose positive $r$ satisfying the 
equation above for $r^{2}$ and let $s=\gamma / 2 r, t=\delta / 2 r$. Either way we obtain $z$ such that $a=\bar{z} i z$. This proves the theorem for $n=1$.

Now assume that the theorem is true for all $(n-1) \times(n-1)$ skew-hermitian matrices and let $A$ be an invertible $n \times n$ skewhermitian matrix. Without loss of generality we can assume that the top left-hand corner entry $a_{11} \neq 0$. (It is an easy exercise to verify that if $\bar{v}^{t} A v=0$ for all column vectors $v \in \mathbf{H}^{n}$, then $\bar{v}^{t} A w=0$ for all $v, w \in \mathbf{H}^{n}$. This implies that $A=0$. Hence there exists $v \in \mathbf{H}^{n}$ with $\bar{v}^{t} A v \neq 0$. Letting $v$ be the first column of an invertible matrix $P$, we can replace $A$ by $\bar{P}^{t} A P$ which has non-zero top left-hand corner entry.) Choose $w \in \mathbf{H}$ such that $\bar{w} a w=i$. (Put $a=a_{11}$ in the $n=1$ case above and then take $w=z^{-1}$.)

Write

$$
A=\left(\begin{array}{cc}
a_{11} & -\bar{L}^{t} \\
L & N
\end{array}\right),
$$

where $L$ is an $(n-1) \times 1$ matrix, and $N$ an $(n-1) \times(n-1)$ skew-hermitian matrix. Let

$$
P=\left(\begin{array}{cc}
w & a_{11}^{-1} \bar{L}^{t} \\
0 & I_{n-1}
\end{array}\right) .
$$

Then we find that

$$
\bar{P}^{t} A P=\left(\begin{array}{ll}
i & 0 \\
0 & B
\end{array}\right),
$$

with $B=N+L a_{11}^{-1} \bar{L}^{t}$ being an invertible $(n-1) \times(n-1)$ skewhermitian matrix. (Note that $B$ is invertible since $A$ and $P$ are invertible.) By the inductive assumption there is an invertible $(n-1) \times(n-1)$ matrix $Q$ with $\bar{Q}^{t} B Q=i I_{n-1}$. The $n \times n$ matrix

$$
R=\left(\begin{array}{ll}
1 & 0 \\
0 & Q
\end{array}\right)
$$

gives the desired result that $\bar{R}^{t} \bar{P}^{t} A P R=i I_{n}$. 


\section{References}

[B] J. L. Brenner, Matrices of quaternions, Pacific J. Math. 1 (1951), 329335 .

[L] H. C. Lee, Eigenvalues and canonical forms for matrices with quaternion coefficients, Proc. Royal Irish Academy, Section A, 52 (1949), 253-260.

[S] W. Scharlau, Quadratic and Hermitian Forms. Grundlehren der math. Wissenschaften 270. Springer-Verlag: Berlin-Heidelberg-New York, 1985.

D. W. Lewis

Department of Mathematics,

University College Dublin,

Belfield,

Dublin 4,

Ireland.

email: dwlewis@ollamh.ucd.ie 